\title{
Admitting mistakes: ethics says yes, instinct says no
}

$\mathrm{S}$ even Massachusetts hospitals recently announced a collaborative effort called "I'm Sorry" that involves disclosure, apology and an offer of compensation to patients who have experienced adverse outcomes while under care in their institutions. The program, the first of its kind from a major group of North American hospitals, is based on earlier efforts in Michigan and Stanford that pioneered open disclosure and integrated it into patient safety activities that emphasized honesty using risk managers with clinical backgrounds. ${ }^{1}$ While one of the main motivations for these programs is to reduce the cost of medical liability suits, a secondary motive is to improve patients' safety and satisfaction while providing institutional assistance to physicians in cases where error and adverse events occur.

Harmful medical errors cause considerable distress for patients and their families. In addition, the psychological and emotional toll on health care professionals, many of whom were selected for training because of their penchant for perfection, can be considerable. Physicians are taught that when they make a mistake, the right course of action is to attempt to correct the error, to admit it and apologize to the patient. ${ }^{2}$ Apologies are perceived as having "profound healing effects" for both physicians, by reducing feelings of guilt, and patients, by promoting forgiveness and reconciliation.

There is a considerable amount of literature supporting disclosure of errors to patients, including some evidence that doing so is helpful in resolving the issue, and does not lead to an increased chance of legal action or financial penalty. ${ }^{1}$ Despite this evidence that disclosure is not harmful, every instinct tells the practitioner otherwise. Admission of error is embarrassing and may lead to poor evaluation, censure or even termination. In addition to the potential career effects, the psychological toll of committing errors, much less admitting them, can be exacerbated when patients and their families, who are emotionally vulner-

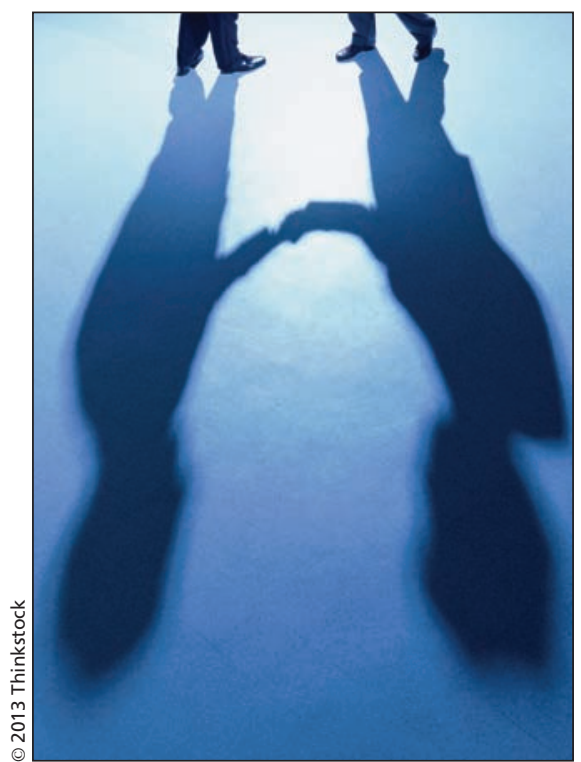

able during times of illness, react with hostility. While delivering bad news is a core skill for physicians, no one looks forward to doing so, especially when he or she is the cause of the bad news.

Under current conditions, it is naïve to expect physicians to voluntarily disclose errors when they perceive that doing so could result in harm to their personal careers. Indeed, trainees in one study reported that they disclosed error to patients in only $24 \%$ of cases. ${ }^{4}$ Attending physicians are no different. ${ }^{5}$ We do believe that it is the patient's right to know when injury-by-error has occurred, and that admission of error is an important component of professionalism in medicine. However, rather than relying on individual, principles-based action, what is needed is a system of support for full disclosure. Perhaps the system changes that took place to respond to needle-stick injuries in health care professionals can serve as a model. The HIV epidemic spurred all health care institutions to develop programs to provide rapid confidential testing, prophylaxis and emotional support when needle-stick injuries occurred. Similar organizational approaches to changing the cultural norm in error disclosure should include moral, professional and legal support for admis- sion of error, as well as appropriate training and role modelling.

Moral support should include ready access to psychological first aid after events occur, and after disclosure and investigation. ${ }^{6}$ Professional support should include clear organizational policy that explicitly prohibits penalties in specific spheres of training and practice. Legal support should include legal privilege for both explanations and apologies that occur during disclosure discussions held with patients (five Canadian provinces have passed "apology legislation" offering such protection $\left.{ }^{3}\right)$. Training should include in-person and online materials as well as just-in-time support for conducting the actual disclosure discussion with the patient and family. Role models should share testimonials and stories from prominent practitioners about their experiences, as well as decisive action taken in real time when incidents occur.

The task of undoing decades of medical culture and generations of evolutionary pressure is formidable and will require institutional approaches that may be based on selfish motives (i.e., reducing liability payments) but induce individual health care professionals to want to admit their mistakes. Focused and concerted efforts will be necessary.

\section{Allan S. Detsky MD PhD}

Institute of Health Policy Management and Evaluation, and Department of Medicine University of Toronto

Toronto, Ont.

Mark O. Baerlocher MD

Department of Radiology

Royal Victoria Hospital

Barrie, Ont.

Albert W. Wu MD MPH

Departments of Health Policy and

Management, and Epidemiology

Johns Hopkins Bloomberg School of

Public Health

Baltimore, Maryland

For references, see Appendix 1, available at www.cmaj.ca/lookup/suppl/doi:10.1503 /cmaj.121187/-/DC1

CMAJ 2013. DOI:10.1503/cmaj.121187 